\title{
Optimal Portfolio Strategy under Rolling Economic Maximum Drawdown Constraints
}

\author{
Xiaojian Yu, ${ }^{1}$ Siyu Xie, ${ }^{2}$ and Weijun $\mathrm{Xu}^{3}$ \\ ${ }^{1}$ School of Economics and Commerce, South China University of Technology, Guangzhou 510006, China \\ ${ }^{2}$ School of Electronic and Information Engineering, South China University of Technology, Guangzhou 510641, China \\ ${ }^{3}$ School of Business Administration, South China University of Technology, Guangzhou 510641, China \\ Correspondence should be addressed to Xiaojian Yu; xjyu@scut.edu.cn
}

Received 27 February 2014; Revised 18 June 2014; Accepted 19 June 2014; Published 10 July 2014

Academic Editor: Pankaj Gupta

Copyright (c) 2014 Xiaojian Yu et al. This is an open access article distributed under the Creative Commons Attribution License, which permits unrestricted use, distribution, and reproduction in any medium, provided the original work is properly cited.

\begin{abstract}
This paper deals with the problem of optimal portfolio strategy under the constraints of rolling economic maximum drawdown. A more practical strategy is developed by using rolling Sharpe ratio in computing the allocation proportion in contrast to existing models. Besides, another novel strategy named "REDP strategy" is further proposed, which replaces the rolling economic drawdown of the portfolio with the rolling economic drawdown of the risky asset. The simulation tests prove that REDP strategy can ensure the portfolio to satisfy the drawdown constraint and outperforms other strategies significantly. An empirical comparison research on the performances of different strategies is carried out by using the 23-year monthly data of SPTR, DJUBS, and 3-month T-bill. The investment cases of single risky asset and two risky assets are both studied in this paper. Empirical results indicate that the REDP strategy successfully controls the maximum drawdown within the given limit and performs best in both return and risk.
\end{abstract}

\section{Introduction}

We study the optimal portfolio strategy under rolling economic maximum drawdown (REDD) constraints. Maximum drawdown refers to the maximum cumulative loss from a peak to a following bottom. The rolling economic maximum drawdown is more reasonable considering the riskfree return after the past maximum [1]. Maximum drawdown is an important risk measurement for money management professionals. It also challenges the clients' financial tolerance. Large drawdown usually leads to fund redemptions. Chekhlov et al. [2] pointed out that a 50\% drawdown in an account is highly intolerable, and an account will be shut down if it suffers from drawdown for more than two years. Therefore, controlling the drawdown is crucial for the fund manager.

After Markowitz [3] initiated the study of mean-variance approach on portfolio selection, expected value and variance are widely accepted as the measures of portfolio return and investment risk. During the recent years, the so-called mean-variance optimization (MVO) problem has advanced much, such as incorporating transaction costs into MVO [4], multiperiod portfolio selection [5], fuzzy investment portfolio selection [6], and extreme value approach in portfolio selection [7]. An important direction of the MVO research is portfolio optimization with risk control. The problem of portfolio optimization subject to drawdown constraints was originally introduced by Grossman and Zhou [1]. In a BlackScholes economy with two securities (one risky and one riskfree), they studied the optimal portfolio problem of a power utility investor, who faces the constraint of losing no more than a fixed fraction of the maximum wealth achieved at any point in time up to that date, by maximizing the long term (asymptotic) growth rate of the expected utility of wealth. Later, Cvitanic and Karatzas [8] generalized this research in a situation in which the multidimensional risky asset price processes are defined by an $n$-dimensional lognormal stochastic differential equation with $n$-dimensional Brownian motion and deterministic coefficients. Specially, the risk-free interest rate is deterministic in the work of Cvitanic and Karatzas [8]. Besides traditional drawdown, Chekhlov et al. [2] formulated the conditional drawdown-at-risk $(\mathrm{CDaR})$ as 
the mean of the worse $(1-\alpha) * 100 \%$ drawdowns and provided the practically stable portfolio using the CDaR measure. Sekine [9] further extended the research to an upside-chance maximization problem of a large deviation probability called dual optimization problem, which links the optimization problem with the drawdown constraint in $[1,8]$ to the standard risk-sensitive-type portfolio optimization problem in Nagai [10] and Kaise and Sheu [11]. Pospisil and Vecer [12] derived probabilistic representations of portfolio sensitivity to maximum drawdown and formulated the investment strategy by taking the forward price of the maximum drawdown into account. A recent work done by Yang and Zhong [13] developed the optimal strategy of $[1,8]$ by proposing a time window to calculate the maximum drawdown, which is called rolling economic drawdown (REDD). Therefore, in their model, the optimal allocation of risky assets is adjusted according to the REDD. The empirical results proved that the optimal strategy based on REDD outperforms the one based on traditional maximum drawdown.

In this paper, our main objective is to do a further research on Yang and Zhong's model by maximizing the expected return as well as controlling the maximum drawdown in order to ensure the loss to stay within an acceptable level. The contribution of this paper is threefold. Firstly, looking back for a rolling window of length $h$, we introduce the "rolling Sharpe ratio" (RSR) to substitute the long term expected Sharpe ratio. Yang and Zhong [13] assumed the Sharpe ratio as a constant parameter, which is not available in practice, in approaching the optimal strategy to control maximum drawdown. We develop a rolling Sharpe ratio strategy in contrast to Yang and Zhong's model. Secondly, we further propose a novel strategy by substituting "rolling economic drawdown of risky asset's prices" $\left(\mathrm{REDD}_{P}\right)$ for "rolling economic drawdown of portfolio" (REDD). When a new investor enters the financial market, what he considers most is the behavior of the risky asset itself. An investor who wants to adjust the portfolio attaches greater importance to the drawdown of the risky asset's price than portfolio wealth. Besides, the models based on the drawdown of the portfolio have a shortage of allocating wealth to different risky assets. Therefore, in this paper, we propose the "rolling economic drawdown of risky asset's prices strategy." We perform the simulation test and prove that the REDP strategy can ensure the portfolio to meet the drawdown constraint and outperforms other strategies. Lastly, we test all of the strategies to the cases of single risky asset and two risky assets with real market data. The correlation of risky assets is also compared. In addition, the empirical results of the strategy with different drawdown constraints are demonstrated in this paper.

The remainder of the paper is organized as follows. Section 2 illustrates the models of different strategies. Section 3 demonstrates the simulation test. The empirical research data and parameters are described in Section 4. Section 5 gives the empirical results and comparison analysis. We conclude and offer the potential future research avenues in Section 6.

\section{The Models of Strategies}

2.1. Problem and Solution. We assume that there is a frictionless financial market with one risk-free asset with a constant return $r$ and $n$ risky assets. The price of each risky asset follows a geometric Brownian motion, modeled by the stochastic equation:

$$
\begin{array}{r}
d P_{i}(t)=P_{i}(t)\left[\mu_{i}(t) d t+\sigma_{i}(t) d Z(t)\right], \quad P_{i}(0)>0, \\
i=1,2, \ldots, n,
\end{array}
$$

where $Z(t)$ is a standard Brownian motion.

Let the initial wealth be $W(0)$ and let the investor's wealth at time $t$ be $W(t)$. The investor invests with a dynamic strategy $\mathbf{x}(t)=\left(x_{1}(t), x_{2}(t), \ldots, x_{n}(t)\right)^{T}$, where $x_{i}(t)$ is the proportion of wealth invested in the $i$ th risky asset. Thus, the corresponding wealth dynamics is governed by

$$
\begin{gathered}
d W(t)=W(t)\left\{\sum_{i=1}^{n} x_{i}(t)\left[\mu_{i}(t) d t+\sigma_{i}(t) d Z(t)\right]\right. \\
\left.+\left[1-\sum_{i=1}^{n} x_{i}(t)\right] r d t\right\}, \\
W_{0}>0 .
\end{gathered}
$$

At time $t$, the investor definitely knows the maximum wealth value since inception. Suppose the maximum wealth value is $W(s)(0 \leq s \leq t)$. If the investor sells out all of the risky assets at time $s$ and puts all of the money in the riskfree asset till time $t$, the wealth would be $W(s) e^{r(t-s)}$. It is the maximum wealth the investor can achieve theoretically. We define it as "economic max" (EM) (Yang and Zhong [13]):

$$
\operatorname{EM}(t)=\max _{0 \leq s \leq t}\left\{W(s) e^{r(t-s)}\right\}
$$

Now, we consider how the investor set a portfolio strategy with economic max. We state the same portfolio optimization problem considered by Grossman and Zhou [1] and suppose the investor invests with a drawdown constraint. Then, the wealth process $W(\cdot)$ should satisfy

$$
W(t) \geq(1-\delta) \operatorname{EM}(t), \quad \forall 0 \leq t \leq \infty,
$$

where $\delta(0<\delta<1)$ is the drawdown limit. If the investor can endure a larger wealth drawdown, he can set a greater $\delta$ as the drawdown limit.

Based on EM, we defined the "economic drawdown" (EDD) as

$$
\operatorname{EDD}(t)=1-\frac{W(t)}{\operatorname{EM}(t)} .
$$

Then, the drawdown constraint becomes

$$
\operatorname{EDD}(t) \leq \delta, \quad \forall 0 \leq t \leq \infty .
$$

Assume the investor has a power utility function with a constant relative risk aversion as

$$
U(W)=\frac{W^{1-A}}{(1-A)},
$$


where $A>0, A \neq 1$, and $W$ is the wealth. A bigger $A$ reflects a stronger risk aversion. Since the drawdown limit and the risk aversion have similar meaning, we set $A=1-\delta$ in this paper. It means that the investor with a stronger risk aversion would set a lower drawdown limit.

The investor's objective is to choose a trading strategy to maximize the long term growth rate of the expected utility of final wealth, which can be denoted as

$$
\varlimsup_{T \rightarrow \infty} \frac{1}{(1-A) T} \ln E[(1-A) U(W(T))] .
$$

Set $\mathbf{x}(t)=\boldsymbol{\pi}(t)(1-(1-\delta) \operatorname{EM}(t) / W(t)), \boldsymbol{\pi}(t)=\left(\pi_{1}(t)\right.$, $\left.\pi_{2}(t), \ldots, \pi_{n}(t)\right)^{T}$. Then, the wealth dynamics is expressed as

$d W(t)$

$$
\begin{aligned}
=W(t)\left\{\sum_{i=1}^{n} \pi_{i}(t)\left(1-\frac{(1-\delta) \operatorname{EM}(t)}{W(t)}\right)\right. \\
\quad \times\left[\mu_{i}(t) d t+\sigma_{i}(t) d Z(t)\right] \\
\left.+\left[1-\sum_{i=1}^{n} \pi_{i}(t)\left(1-\frac{(1-\delta) \operatorname{EM}(t)}{W(t)}\right)\right] r d t\right\}, \\
W_{0}>0 .
\end{aligned}
$$

According to the results of Cvitanic and Karatzas [8] and letting $A=1-\delta$, the optimal portfolio strategy is given by

$$
\boldsymbol{\pi}(t)=\left[\boldsymbol{\sigma}(t)^{-1} \boldsymbol{\mu}(t)\right]^{T} \boldsymbol{\sigma}(t)^{-1} \frac{1}{1-\delta^{2}},
$$

where $\boldsymbol{\sigma}(t)$ is the volatility matrix $\left(\Omega(t)=\boldsymbol{\sigma}(t) \boldsymbol{\sigma}(t)^{T} ; \Omega(t)\right.$ is the covariance matrix) and $\boldsymbol{\mu}(t)$ is the return drift vector that $\mu_{i}(t)=b_{i}(t)-r$.

Then, the proportion vector of wealth of the risky assets is

$$
\begin{aligned}
\mathbf{x}(t) & =\left[\boldsymbol{\sigma}(t)^{-1} \boldsymbol{\mu}(t)\right]^{T} \boldsymbol{\sigma}(t)^{-1} \frac{1}{1-\delta^{2}}\left(1-\frac{(1-\delta) \operatorname{EM}(t)}{W(t)}\right) \\
& =\left[\boldsymbol{\sigma}(t)^{-1} \boldsymbol{\mu}(t)\right]^{T} \boldsymbol{\sigma}(t)^{-1} \frac{1}{1-\delta^{2}}\left(\frac{\delta-\operatorname{EDD}(t)}{1-\operatorname{EDD}(t)}\right) .
\end{aligned}
$$

2.2. Grossman and Zhou's Strategy. Grossman and Zhou [1] considered a market with only one risky asset and set $b(t)=b$ and $\sigma(t)=\sigma$. With the short-sale constraint and letting $A=$ $1-\delta$, the proportion of wealth allocated to a single risky asset at time $t$ is

$$
x(t)=\max \left\{0, \frac{b-r}{\sigma^{2}\left(1-\delta^{2}\right)}\left(\frac{\delta-\operatorname{EDD}(t)}{1-\operatorname{EDD}(t)}\right)\right\} .
$$

When practising this strategy, we have to adopt the estimated parameters $b$ and $\sigma$. Using the MLE method to estimate the geometric Brownian motion (1) with $b(t)=b$ and $\sigma(t)=\sigma$, we obtain $b=R+0.5 \sigma^{2}$, where $R$ and $\sigma$ are sample mean and sample volatility of the risky asset.
Then, in practice, the proportion of wealth allocated to a single risky asset is

$$
x(t)=\max \left\{0,\left(\frac{R-r}{\sigma^{2}}+\frac{1}{2}\right) \frac{1}{1-\delta^{2}}\left(\frac{\delta-\operatorname{EDD}(t)}{1-\operatorname{EDD}(t)}\right)\right\} .
$$

2.3. Yang and Zhong's Strategy. Yang and Zhong [13] stated that EDD in Grossman and Zhou's model reflects an idealistic mental accounting from sophisticated investors. Investors may enter at different time or be forced to exit. A portfolio inception difference exists among investors. Besides, not all investors have a memory since time zero. Therefore, they modified the "economic max" (EM) by looking back at portfolio wealth history with a constant rolling window of length $H$ and defined a new variable called "rolling economic max" (REM):

$$
\operatorname{REM}(t, H)=\max _{t-H \leq s \leq t}\left\{W(s) e^{r(t-s)}\right\} .
$$

Meanwhile, the "rolling economic drawdown" (REDD) is defined by

$$
\operatorname{REDD}(t, H)=1-\frac{W(t)}{\operatorname{REM}(t, H)}
$$

Based on REDD and REM, Yang and Zhong [13] proposed a "rolling economic drawdown-controlled optimal portfolio strategy" ("YZ strategy" for short) by substituting the REDD for EDD in (12), so that the long term wealth growth rate is maximized under a dynamic constraint:

$$
\operatorname{REDD}(t, H) \leq \delta, \quad \forall 0 \leq t \leq \infty .
$$

Yang and Zhong [13] defined the $S=(R-r) / \sigma$ as the long term expected Sharpe ratio of the risky asset, where $R$ and $\sigma$ are long term expected return and volatility. Therefore, the proportion allocated to a single risky asset with the short-sale constraint in practice is

$$
x^{\mathrm{YZ}}(t)=\max \left\{0,\left(\frac{S}{\sigma}+\frac{1}{2}\right) \frac{1}{1-\delta^{2}}\left(\frac{\delta-\operatorname{REDD}(t, H)}{1-\operatorname{REDD}(t, H)}\right)\right\} .
$$

Comparing (5) with (15), it is obvious that REDD is lower than EDD. It means that, betting on a market rebound, more capital will be allocated to risky asset in order to improve portfolio return.

According to (11), the allocation proportion of multiple risky assets without short-sale constraint in $Y Z$ strategy is given by

$$
\mathbf{x}^{\mathrm{YZ}}(t)^{T}=\left[\boldsymbol{\sigma}^{-1} \boldsymbol{\mu}\right]^{T} \boldsymbol{\sigma}^{-1} \frac{1}{1-\delta^{2}}\left(\frac{\delta-\operatorname{REDD}(t, H)}{1-\operatorname{REDD}(t, H)}\right),
$$

where $\sigma$ is the volatility matrix $\left(\Omega=\sigma \sigma^{T} ; \Omega\right.$ is the covariance matrix) and $\boldsymbol{\mu}$ is the return drift vector that $\mu_{i}=R_{i}-r+\sigma_{i}^{2} / 2$.

For two risky assets, the proportion vector with the shortsale constraint is 


$$
\left[\begin{array}{c}
x_{1}^{\mathrm{YZ}}(t) \\
x_{2}^{\mathrm{YZ}}(t)
\end{array}\right]=\left[\begin{array}{c}
\max \left(0, \frac{S_{1}+0.5 \sigma_{1}-\rho\left(S_{2}+0.5 \sigma_{2}\right)}{\left(1-\rho^{2}\right) \sigma_{1}\left(1-\delta^{2}\right)}\left(\frac{\delta-\operatorname{REDD}(t, H)}{1-\operatorname{REDD}(t, H)}\right)\right) \\
\max \left(0, \frac{S_{2}+0.5 \sigma_{2}-\rho\left(S_{1}+0.5 \sigma_{1}\right)}{\left(1-\rho^{2}\right) \sigma_{2}\left(1-\delta^{2}\right)}\left(\frac{\delta-\operatorname{REDD}(t, H)}{1-\operatorname{REDD}(t, H)}\right)\right)
\end{array}\right] .
$$

In empirical research, Yang and Zhong [13] estimated the long term expected Sharpe ratio $S$ and correlation coefficient $\rho$ with the whole sample data and used the estimated volatility $\sigma(t, h)$ of the time interval $[t-h, t]$ for $\sigma$ in (17) and (19).

2.4. Rolling Sharpe Ratio Strategy. YZ strategy is more practicable comparing to Grossman and Zhou's strategy. However, it employs a long term expected Sharpe ratio to calculate the allocation proportion. In the back testing, Yang and Zhong [13] used the average Sharpe ratio of the whole past test period, which is not available in practice. This is because the investor could not obtain the data that happens in the future. From the practicable point of view, in this paper, we propose the "rolling Sharpe ratio" (RSR) by looking back for a rolling window of length $h$, to substitute for the long term expected Sharpe ratio.

We modify the $Y Z$ strategy by substitute $\mathrm{RS}(t, h)$ for $S$ in (17). We also use the estimated volatility $\sigma(t, h)$ for $\sigma$. Then, the proportion allocated to a single risky asset with the shortsale constraint is

$$
\begin{aligned}
& x^{\mathrm{RS}}(t) \\
& =\max \left\{0,\left(\frac{\mathrm{RS}(t, h)}{\sigma(t, h)}+\frac{1}{2}\right) \frac{1}{1-\delta^{2}}\left(\frac{\delta-\operatorname{REDD}(t, H)}{1-\operatorname{REDD}(t, H)}\right)\right\} .
\end{aligned}
$$

We name it rolling Sharpe ratio strategy (" $R S$ strategy" for short). Here, we assume that $\operatorname{RS}(t, h)$ and the volatility $\sigma(t, h)$ are estimated with the same time interval $[t-h, t]$.

For multiple risky assets, the allocation proportion vector without short-sale constraint is

$$
\begin{aligned}
& \mathbf{x}^{\mathrm{RS}}(t)^{T} \\
& =\left[\boldsymbol{\sigma}(t, h)^{-1} \boldsymbol{\mu}(t, h)\right]^{T} \boldsymbol{\sigma}(t, h)^{-1} \frac{1}{1-\delta^{2}}\left(\frac{\delta-\operatorname{REDD}(t, H)}{1-\operatorname{REDD}(t, H)}\right),
\end{aligned}
$$

where $\boldsymbol{\sigma}(t, h)$ is the volatility matrix $\left(\Omega(t, h)=\boldsymbol{\sigma}(t, h) \boldsymbol{\sigma}(t, h)^{T}\right.$; $\Omega(t, h)$ is the covariance matrix in $[t-h, t])$ and $\boldsymbol{\mu}(t, h)$ is the return drift vector that $\mu_{i}(t, h)=R_{i}(t, h)-r+\sigma_{i}^{2}(t, h) / 2$.

For two risky assets, the proportion vector with the shortsale constraint is

$$
\left[\begin{array}{l}
x_{1}^{\mathrm{RS}}(t) \\
x_{2}^{\mathrm{RS}}(t)
\end{array}\right]=\left[\begin{array}{l}
\max \left(0, \frac{\mathrm{RS}_{1}(t, h)+0.5 \sigma_{1}(t, h)-\rho(t, h)\left(\mathrm{RS}_{2}(t, h)+0.5 \sigma_{2}(t, h)\right)}{\left(1-\rho(t, h)^{2}\right) \sigma_{1}(t, h)\left(1-\delta^{2}\right)}\left(\frac{\delta-\operatorname{REDD}(t, H)}{1-\operatorname{REDD}(t, H)}\right)\right) \\
\max \left(0, \frac{\mathrm{RS}_{2}(t, h)+0.5 \sigma_{2}(t, h)-\rho(t, h)\left(\mathrm{RS}_{1}(t, h)+0.5 \sigma_{1}(t, h)\right)}{\left(1-\rho(t, h)^{2}\right) \sigma_{2}(t, h)\left(1-\delta^{2}\right)}\left(\frac{\delta-\operatorname{REDD}(t, H)}{1-\operatorname{REDD}(t, H)}\right)\right)
\end{array}\right]
$$

2.5. Rolling Economic Drawdown of Risky Asset's Prices Strategy. All of the models presented above are based on the drawdown of the portfolio. However, when a new investor starts to invest in the asset, what he cares about most is the behavior of the risky asset itself. Likewise, the investor pays more attention to the drawdown of the risky asset's prices than portfolio wealth when adjusting the portfolio. Therefore, in contrast with REM, we propose the "rolling economic max of risky asset's prices" $\left(\mathrm{REM}_{P}\right)$ by looking back at the risky asset's prices for a rolling window of length $H$ as follows:

$$
\operatorname{REM}_{P}(t, H)=\max _{t-H \leq s \leq t}\left\{P(s) e^{r(t-s)}\right\} .
$$

Similarly, the "rolling economic drawdown of risky asset's prices" $\left(\mathrm{REDD}_{P}\right)$ is defined by

$$
\operatorname{REDD}_{P}(t, H)=1-\frac{P(t)}{\operatorname{REM}_{P}(t, H)} .
$$

In this sense, $\operatorname{REDD}_{P}$ could be explained as a potential maximum lost ratio of a risky asset during time $t$ to $H$. We explain the advantage of $\operatorname{REDD}_{P}$ over REDD when the investor builds the strategy as follows.

Firstly, the $\mathrm{REDD}_{P}$ is a useful risk measure of the risky asset. When the Sharpe ratio and volatility are the same for different assets, the $\operatorname{REDD}_{P}$ can be used to distinguish the risk. For a two-risky-asset market, the invest proportions of the risky asset in YZ strategy and RS strategy both depend on the REDD, which is the rolling economic drawdown of the portfolio. If two assets have different drawdown as $\mathrm{REDD}_{P}$ while Sharpe ratio and volatility are the same, these strategies would allocate the same wealth to these assets. However, actually, the investor should make a difference between them in allocation. Hence, when considering the individual risk, the $\mathrm{REDD}_{P}$ is more effective than REDD. 
Secondly, substituting the $\mathrm{REDD}_{P}$ of each risky asset for REDD will produce a more flexible strategy especially for multiple assets investment. It is obvious that the invest proportion vector of the risky asset embeds the drawdown constraint with $(\delta-$ REDD $) /(1-$ REDD). It has the same impact on all of the risky assets that would cause an unreasonable investment. For instance, under the short-sale constraint, if $\mathrm{REDD} \geq \delta$, the allocation proportion vector is equal to zero. It means that investor should sell out all of the risky assets even the profitable risky asset. To overcome this shortage, we substitute the $\mathrm{REDD}_{P}$ of each risky asset for REDD in $(\delta-\mathrm{REDD}) /(1-\mathrm{REDD})$. The allocation of each risky asset will adapt its own $\operatorname{REDD}_{P}$.

Thirdly, the Monte Carlo simulation test in the next section proves that adjusting the invest proportion of the risky asset with $\mathrm{REDD}_{P}$ not only ensures the portfolio to satisfy the drawdown constraint but also achieves better performance.

Based on the above analysis, we substitute the $\operatorname{REDD}_{P}$ of each risky asset for REDD in (20) (22) and obtain a new strategy named "rolling economic drawdown of risky asset's prices strategy" (REDP strategy). The purpose of this strategy is to control the drawdown risk as YZ strategy and RS strategy and to seek a higher return.
For a single risky asset, the proportion allocated with the short-sale constraint is

$$
\begin{aligned}
& x^{\operatorname{REDP}}(t) \\
& =\max \left\{0,\left(\frac{\operatorname{RS}(t, h) / \sigma(t, h)+0.5}{1-\delta^{2}}\right)\left(\frac{\delta-\operatorname{REDD}_{P}(t, H)}{1-\operatorname{REDD}_{P}(t, H)}\right)\right\} .
\end{aligned}
$$

For multiple risky assets, the allocation proportion vector without the short-sale constraint is given by

$$
\mathbf{x}^{\mathrm{REDP}}(t)^{T}=\left[\boldsymbol{\sigma}(t, h)^{-1} \boldsymbol{\mu}(t, h)\right]^{T} \boldsymbol{\sigma}(t, h)^{-1} \operatorname{diag}(\mathbf{Y}),
$$

where $\boldsymbol{\sigma}(t, h)$ and $\boldsymbol{\mu}(t, h)$ are defined the same as (21) and $\mathbf{Y}=$ $\left(y_{1}, y_{2}, \ldots, y_{n}\right)^{T}$ is a vector with $y_{i}$ defined as follows:

$$
y_{i}=\frac{1}{1-\delta^{2}}\left(\frac{\delta-\operatorname{REDD}_{P, i}(t, H)}{1-\operatorname{REDD}_{P, i}(t, H)}\right) .
$$

As for two risky assets with the short-sale constraint, the proportion vector is

$$
\left[\begin{array}{l}
x_{1}^{\mathrm{REDP}}(t) \\
x_{2}^{\mathrm{REDP}}(t)
\end{array}\right]=\left[\begin{array}{c}
\max \left(0, \frac{\mathrm{RS}_{1}(t, h)+0.5 \sigma_{1}(t, h)-\rho(t, h)\left(\mathrm{RS}_{2}(t, h)+0.5 \sigma_{2}(t, h)\right)}{\left(1-\rho(t, h)^{2}\right) \sigma_{1}(t, h)} y_{1}\right) \\
\max \left(0, \frac{\mathrm{RS}_{2}(t, h)+0.5 \sigma_{2}(t, h)-\rho(t, h)\left(\mathrm{RS}_{1}(t, h)+0.5 \sigma_{1}(t, h)\right)}{\left(1-\rho(t, h)^{2}\right) \sigma_{2}(t, h)} y_{2}\right)
\end{array}\right] .
$$

Comparing to $Y Z$ strategy and RS strategy, the REDP strategy allocates the wealth based on the $\mathrm{REDD}_{P}$ of each risky asset instead of the REDD of the portfolio. We have Corollaries 1 and 2 .

Corollary 1. If two risky assets have the same Sharpe ratios and volatility, the bigger the $R E D D_{P}$ is, the less the money allocated to the risky asset is.

The $R E D D_{P} s$ play a key role in allocation when other parameter values are the same in (28). Since $f(x)=(\delta-x) /(1-$ x) $(0<\delta<1)$ is a monotonic decreasing function, a big $R E D D_{P, i}$ would cause a small $y_{i}$. Thus, $x_{i}^{\text {REDPS }}$ will be a small one. Such consequence leads to a conservative invest-ment strategy on more risky asset according to its history behavior.

Corollary 2. If the REDD $D_{P}$ of the risky asset is bigger than the REDD of the portfolio, less money would be allocated to the risky asset in contrast to the $Y Z$ strategy and RS strategy.

It is hard to use mathematical derivation to prove whether REDP strategy ensures the portfolio to satisfy the drawdown constraint or whether REDP strategy outperforms other strategies. Therefore, we employ the simulation method to demonstrate it in the next section.

\section{Simulation Test}

In this section, we demonstrate the simulation in two cases: the single risky asset case and the two risky assets' case. The purpose of the simulation is to prove that REDP strategy, YZ strategy, and RS strategy can ensure the portfolio to satisfy the drawdown constraint and to show the outperformance of REDP strategy.

3.1. Single Risky Asset Case. We first study a market with single risky asset. The risky asset follows the motion modeled by

$$
d P(t)=P(t)[\mu d t+\sigma d Z(t)], \quad P(0)>0 .
$$

We simulate the price path with 500 months. The setting detail of the simulation is as follows: the time step length $\Delta t=1 / 12$ (1 month), annual return $\mu=3.98 \%$, annual volatility $\sigma=14 \%$, annual risk-free rate $r=3 \%$, and initial price $P_{0}=100$. We simulate 5000 price paths in total.

For each simulation, we apply the REDP strategy, YZ strategy, and $R S$ strategy to adjust the portfolio with a drawdown limit equal to $1 / 3$. We find that the $Y Z$ strategy will not be performed because of the big negative Sharpe ratio. There are 1426 simulations in which the YZ strategy is not performed. In order to compare these three strategies, we exclude these simulations. The statistical results of these strategies in 3574 simulations are listed in Table 1. 
TABLE 1: Performance statistics of single risky asset simulation case.

\begin{tabular}{lccc}
\hline & YZ strategy & RS strategy & REDP strategy \\
\hline Max & & & \\
Max REDD & $30.372 \%$ & $22.066 \%$ & $23.439 \%$ \\
Maximum drawdown & $47.138 \%$ & $22.746 \%$ & $28.961 \%$ \\
Annualized return & $11.958 \%$ & $6.679 \%$ & $7.663 \%$ \\
Annualized return S.E. & $14.471 \%$ & $6.597 \%$ & $0.226 \%$ \\
Sharpe ratio & 0.654 & 0.566 & 20.266 \\
Final wealth & 99.786 & 13.941 & \\
Mean & & & $9.581 \%$ \\
Max REDD & $11.641 \%$ & $12.184 \%$ & $8.301 \%$ \\
Maximum drawdown & $12.865 \%$ & $11.131 \%$ & $4.425 \%$ \\
Annualized return & $3.952 \%$ & $3.314 \%$ & $4.895 \%$ \\
Annualized return S.E. & $5.449 \%$ & $5.286 \%$ & 0.276 \\
Sharpe ratio & 0.098 & 0.046 & 6.064 \\
Final wealth & 5.625 & 3.934 & \\
\hline
\end{tabular}

Note: $\Delta t=1 / 12, P_{0}=100, \mu=3.98 \%, \sigma=14 \%, r=3 \%$, and price path length $=500$ months. Also $\delta=1 / 3.3574$ simulations. Final wealth refers to the return at the end of the investment period if one dollar was invested at the beginning of the period.

TABLE 2: Performance test of single risky asset simulation case.

\begin{tabular}{|c|c|c|c|c|c|c|}
\hline & Max REDD & Maximum drawdown & Annualized return & Annualized return S.E. & Sharpe ratio & Final wealth \\
\hline $\operatorname{Mean}\left(S_{R E D P-R S}\right)$ & $-2.603 \%$ & $-2.830 \%$ & $1.111 \%$ & $-0.392 \%$ & 0.230 & 2.130 \\
\hline$P$ value & 0.000 & 0.000 & 0.000 & 0.000 & 0.000 & 0.000 \\
\hline $\operatorname{Mean}\left(S_{R E P D-Y Z}\right)$ & $-2.061 \%$ & $-4.565 \%$ & $0.473 \%$ & $-0.555 \%$ & 0.178 & 0.440 \\
\hline$P$ value & 0.000 & 0.000 & 0.000 & 0.000 & 0.000 & 0.000 \\
\hline
\end{tabular}

Note: the null hypothesis is as follows: the performance of REDP strategy is the same as YZ strategy and RS strategy.

In Table 1 , the max value of the max REDD of each strategy is smaller than the drawdown limit $(\delta=1 / 3)$. It means that all of the strategies succeed in controlling the portfolio within the given loss. Although the REDP strategy substitutes the $\mathrm{REDD}_{P}$ for REDD, it can also satisfy the drawdown constraint.

Table 1 also clearly shows the mean performance of each strategy. It indicates that, among these three strategies, on average, REDP strategy has the smallest max REDD, maximum drawdown and volatility (annualized return S.E.), and the highest return, Sharpe ratio, and final wealth.

In order to test whether the REDP strategy outperforms the other two, we perform the $t$-test. The null hypothesis is as follows. The performance of REDP strategy is the same as $Y Z$ strategy and RS strategy. The statistics are calculated by

$$
\begin{gathered}
S_{\mathrm{REDP}-\mathrm{RS}}=S_{\mathrm{REDP}, i}-S_{\mathrm{RS}, i} \\
S_{\mathrm{REDP}-\mathrm{YZ}}=S_{\mathrm{REDP}, i}-S_{\mathrm{YZ}, i},
\end{gathered}
$$

where $S_{i}$ is one of the performance values (max REDD, maximum drawdown, annualized return, annualized return S.E., Sharpe ratio, and final wealth). The average performance difference and test results are exhibited in Table 2.

The test results in Table 2 reject the hypothesis at $1 \%$ level, which means the performance of REDP strategy differs from $Y Z$ strategy and $R S$ strategy significantly. The value of $\operatorname{Mean}\left(S_{\text {REDP-RS }}\right)$ (Mean $\left.\left(S_{\text {REPD-YZ }}\right)\right)$ is the average difference between REDP strategy and RS strategy ( $Y Z$ strategy). It is obvious that the REDP strategy performs better than $R S$ strategy and $Y Z$ strategy in controlling risk and producing return.

3.2. Two Risky Assets Case. For a market with two risky assets, we also assume both risky assets follow the motion modeled by (28) and simulate the price path of each asset with 500 months. The detailed simulation setting is as follows: $\Delta t=$ $1 / 12$, annual return $\mu_{1}=3.98 \%, \mu_{2}=2.48 \%$, annual volatility $\sigma_{1}=\sigma_{2}=14 \%$, correlation $\rho=0.1$, annual risk-free rate $r=3 \%$, and initial price $P_{1,0}=P_{2,0}=100$. Likewise, we conduct 5000 simulations in total.

We test the REDP strategy, YZ strategy, and RS strategy with the same drawdown limit $1 / 3$ and consider the correlation effect. The statistical results of these strategies in 5000 simulations are showed in Table 3.

In Table 3, all of the max values of the max REDD are smaller than $1 / 3$. We can also conclude that all of the strategies can control the portfolio within the drawdown limit. Therefore, the simulation test proves that REDP strategy satisfies the drawdown constraint. Obviously, the results of mean performance of each strategy indicate that REDP strategy produces the highest return and controls the risk very well.

We follow the same procedure to test the same null hypothesis described in Section 3.1. The statistics are also calculated by (30). The results are exhibited in Table 4. 
TABLE 3: Performance statistics of two-risky-asset simulation case.

\begin{tabular}{lccc}
\hline & YZ strategy & RS strategy & REDP strategy \\
\hline Max & & & $19.598 \%$ \\
Max REDD & $22.529 \%$ & $21.724 \%$ & $44.005 \%$ \\
Maximum drawdown & $38.701 \%$ & $72.348 \%$ & $7.899 \%$ \\
Annualized return & $8.984 \%$ & $5.882 \%$ & $8.087 \%$ \\
Annualized return S.E. & $10.201 \%$ & $8.367 \%$ & 0.973 \\
Sharpe ratio & 0.888 & 0.699 & 22.155 \\
Final wealth & 33.308 & 10.270 & $12.029 \%$ \\
Mean & & & $18.137 \%$ \\
Max REDD & $12.039 \%$ & $14.566 \%$ & $2.803 \%$ \\
Maximum drawdown & $19.699 \%$ & $30.211 \%$ & $6.649 \%$ \\
Annualized return & $1.909 \%$ & $1.123 \%$ & 0.411 \\
Annualized return S.E. & $5.528 \%$ & $6.970 \%$ & 3.412 \\
Sharpe ratio & 0.304 & 0.151 & 1.759 \\
Final wealth & 2.515 & & \\
\hline
\end{tabular}

Note: $\Delta t=1 / 12, P_{1,0}=P_{2,0}=100, \mu_{1}=3.98 \%, \mu_{2}=2.48 \%, \sigma_{1}=\sigma_{2}=14 \%, r=3 \%$, and price path length $=500$ months. Also $\delta=1 / 3.5000$ simulations. Final wealth refers to the return at the end of the investment period if one dollar was invested at the beginning of the period.

TABle 4: Performance test of two-risky-asset simulation case.

\begin{tabular}{|c|c|c|c|c|c|c|}
\hline & Max REDD & Maximum drawdown & Annualized return & Annualized return S.E. & Sharpe ratio & Final wealth \\
\hline $\operatorname{Mean}\left(S_{R E P D-R S}\right)$ & $-2.536 \%$ & $-12.074 \%$ & $1.679 \%$ & $-0.320 \%$ & 0.259 & 1.653 \\
\hline$P$ value & 0.000 & 0.000 & 0.000 & 0.000 & 0.000 & 0.000 \\
\hline $\operatorname{Mean}\left(S_{R E P D-Y Z}\right)$ & $-0.010 \%$ & $-1.562 \%$ & $0.894 \%$ & $1.121 \%$ & 0.107 & 0.899 \\
\hline$P$ value & 0.838 & 0.000 & 0.000 & 0.000 & 0.000 & 0.000 \\
\hline
\end{tabular}

Note: the null hypothesis is as follows: the performance of REDP strategy is the same as YZ strategy and RS strategy.

The results in Table 4 reject the hypothesis at $1 \%$ level except for the hypothesis that the max REDD of REDP strategy is different from YZ strategy at $10 \%$ level. We can also conclude that the performance of REDP strategy is significantly different from YZ strategy and RS strategy.

The comparison results of average performance difference are a little different from single risky asset case. In two risky assets' case, the volatility of REDP strategy is bigger than $Y Z$ strategy, and the max REDD of REDP strategy is very close. However, the return, Sharpe ratio, and final wealth of REDP strategy are significantly greater than $Y Z$ strategy. In addition, all of the values of Mean $\left(S_{\text {REDP-RS }}\right)$ prove that the $R E D P$ strategy outperforms $R S$ strategy at risk and return.

All in all, the simulation tests prove that REDP strategy can ensure the portfolio to satisfy the drawdown constraint and outperforms other strategies. In the next section, we use the real financial data to illustrate that the REDP strategy can control the drawdown risk and outperforms the other two strategies.

\section{Empirical Data and Parameters}

In Yang and Zhong's [13] empirical research, they proved that monthly rebalance is better than the daily and weekly ones. In this paper, we refer to their means and use monthly data to rebalance the allocation between the risky assets and risk-free asset. To compare the performance of different strategies empirically, we use the 23-year monthly data of US stock market and commodity market from January 1991 to December 2013. Standard \& Poor's 500 total return index (SPTR) and Dow-Jones UBS-commodity total return index (DJUBS) are used for the risky assets. The risk-free asset is the 3-month US treasury-bill (3-month T-bill).

Since a frequent trading will cause transaction cost, we set transaction cost to be $0.3 \%$ throughout. Although the length of the rolling time window of REDD and the rolling time window of Sharpe ratio and correlation coefficient may differ, we assume that the lengths are the same for simplicity. In order to better reflect the fluctuation of the market, we set the length of the rolling time windows to be $H=h=1$ year, which is shorter than the length of market cycle.

In our empirical research, we forbid any kind of short selling. Thus, the proportion of wealth invested in both risky assets and risk-free asset is nonnegative. To ensure the total capital exposure is no more than $100 \%$, the allocation weights are normalized.

As investors have difference risk preference, we test 4 different drawdown limit values, $1 / 3,0.25,0.2$, and 0.15 . However, in the comparison of different strategies, we set $\delta=1 / 3$ only.

For Sharpe ratio, the strategies differ in its calculation. In YZ strategy, the Sharpe ratio of the risky asset is a constant parameter estimated from the in-sample process. In $R S$ strategy and REDP strategy, the Sharpe ratio and volatility 
TABLE 5: Performance statistics of single risky asset investment.

\begin{tabular}{|c|c|c|c|c|c|c|c|c|}
\hline \multirow{2}{*}{ Statistics } & \multicolumn{4}{|c|}{ SPTR } & \multicolumn{4}{|c|}{ DJUBS } \\
\hline & Historical data & YZ strategy & RS strategy & REDP strategy & Historical data & YZ strategy & RS strategy & REDP strategy \\
\hline Max REDD & $47.90 \%$ & $27.94 \%$ & $9.66 \%$ & $7.99 \%$ & $54.79 \%$ & $3.28 \%$ & $18.69 \%$ & $6.07 \%$ \\
\hline Mean REDD & $6.51 \%$ & $4.98 \%$ & $2.40 \%$ & $1.80 \%$ & $9.16 \%$ & $0.21 \%$ & $3.03 \%$ & $1.95 \%$ \\
\hline Maximum drawdown & $52.56 \%$ & $31.33 \%$ & $9.64 \%$ & $8.17 \%$ & $54.52 \%$ & $2.74 \%$ & $18.08 \%$ & $7.31 \%$ \\
\hline Annualized return & $7.13 \%$ & $6.83 \%$ & $6.23 \%$ & $7.27 \%$ & $1.50 \%$ & $2.81 \%$ & $3.00 \%$ & $4.50 \%$ \\
\hline Annualized return S.E. & $14.65 \%$ & $10.67 \%$ & $6.33 \%$ & $5.98 \%$ & $14.96 \%$ & $0.71 \%$ & $5.49 \%$ & $4.87 \%$ \\
\hline Sharpe ratio & 0.2891 & 0.3693 & 0.5272 & 0.7324 & -0.0929 & -0.1104 & 0.0202 & 0.3301 \\
\hline Final wealth & 4.4787 & 4.2805 & 3.7793 & 4.685 & 1.3906 & 1.8414 & 1.9173 & 2.6341 \\
\hline
\end{tabular}

Note: since the length of the rolling time window is one year, the period conducting the strategy is from March 1992 to December 2013.

are estimated by forward looking at the risky asset history for a time window $H$. The REDD of the YZ strategy and the REDD of the RS strategy are both computed with the history data of portfolio wealth. But for the REDP strategy, the $\mathrm{REDD}_{P}$ is computed with the history data of risky asset.

\section{Empirical Analysis}

In this section, the empirical analysis is emphasized on the comparison between the three strategies ( $Y Z$ strategy, RS strategy, and REDP strategy). We study the cases of both single risky asset and two risky assets. Since $Y Z$ strategy assumed that multiple risky assets are uncorrelated, the correlation coefficient $\rho$ between the two risky assets is neglected. In order to analyze the impact of correlation, this paper further compares the investment performance of the situation with and without considering correlation of risky assets.

5.1. Single Risky Asset. For single risky asset analysis, we invest in the SPTR and DJUBS individually. In both cases, the risk-free asset, 3-month T-bill, is the same. The monthly rebalance is made between the single risky asset (SPTR or DJUBS) and the risk-free asset (3-month T-bill). The investment performances of the three strategies ( $Y Z$ strategy, $R S$ strategy, and REDP strategy) are calculated, respectively. Set the drawdown limit $\delta=1 / 3$; the statistic results of different strategies are exhibited in Table 5.

In Table 5, although the max REDD of the risky asset is high $(47.90 \%$ for SPTR and $54.79 \%$ for DJUBS), all of the max REDD and maximum drawdown of the three strategies are below the drawdown limit $1 / 3$. It means that the strategy models we developed in this paper are suitable for risk control.

For investing in the risky asset SPTR, the REDP strategy achieves higher annualized return, larger Sharpe ratio, lower max REDD, and smaller annualized return standard error than $Y Z$ strategy and $R S$ strategy. Final wealth refers to the return at the end of the investment period if one dollar was invested at the beginning of the period. Both annualized return and final wealth of $Y Z$ strategy and RS strategy are lower than the risky asset. It means that $Y Z$ strategy and $R S$ strategy can reduce the investment risk but sacrifice the profit.
In contrast, the return of the REDP strategy is much higher and more stable, while the risk is reduced.

For investing in the risky asset DJUBS, even though the max REDD of $Y Z$ strategy is slightly lower than the REDP strategy, $Y Z$ strategy suffers from a negative Sharpe ratio. Under this circumstance, investors would rather invest in the risk-free asset than invest in the risky asset, because the low Sharpe ratio indicates that the return of the risky asset is worse than that of the risk-free one. Besides, the annualized return of the REDP strategy is higher than YZ strategy and RS strategy.

The results of different risky assets above consistently verify that our REDP strategy is the best among the three strategies. The REDP strategy can both lower the risk by controlling the max REDD and maximum drawdown within the target level $\delta$ and enhance the return with less volatility.

5.2. Two Risky Assets. In addition to the single risky asset, we extend the empirical research to the case of two risky assets. Once again, the risky assets are SPTR and DJUBS, and the risk-free asset is 3-month T-bill. We also do a monthly rebalance between the two risky assets according to the investment weight calculated from the models, and the rest of the capital is invested in the risk-free asset.

Since the correlation coefficient $\rho$ between the risky assets may have an impact on the final results, in this section, we compare the empirical results of the three strategies with and without considering correlation. For the uncorrelated case, we neglect the correlation coefficient $\rho$ between the two risky assets; and for the correlated case, the correlation coefficient is included in the models.

In $Y Z$ strategy, the correlation coefficient $\rho$ is a constant parameter estimated from the 23-year test data. In the $R S$ strategy and the REDP strategy, the parameter $\rho$ is estimated by forward looking at the historical data for a one-year rolling time window. The calculation process is similar to that of the single risky asset case. Table 6 shows the empirical results with the drawdown limit $\delta=1 / 3$.

With drawdown limit $\delta=1 / 3$, all of the max REDD and maximum drawdown in Table 6 stay within the target level. Among the three strategies, the max REDD and maximum drawdown are the lowest in the REDP strategy, while annualized return and Sharpe ratio are the highest. It is obvious that our REDP strategy achieves the best performance, which 
TABLE 6: Performance statistics of two risky assets' investment.

\begin{tabular}{|c|c|c|c|c|c|c|}
\hline \multirow{2}{*}{ Statistics } & \multicolumn{3}{|c|}{ Without correlation coefficient } & \multicolumn{3}{|c|}{ With correlation coefficient } \\
\hline & YZ strategy & RS strategy & REDP strategy & YZ strategy & RS strategy & REDP strategy \\
\hline Max REDD & $28.40 \%$ & $18.87 \%$ & $11.87 \%$ & $19.93 \%$ & $23.20 \%$ & $7.96 \%$ \\
\hline Mean REDD & $5.01 \%$ & $3.56 \%$ & $2.24 \%$ & $2.59 \%$ & $3.33 \%$ & $1.99 \%$ \\
\hline Maximum drawdown & $31.86 \%$ & $19.97 \%$ & $11.85 \%$ & $21.27 \%$ & $25.47 \%$ & $8.13 \%$ \\
\hline Annualized return & $6.83 \%$ & $5.81 \%$ & $8.28 \%$ & $4.80 \%$ & $5.36 \%$ & $7.64 \%$ \\
\hline Annualized return S.E. & $10.70 \%$ & $8.23 \%$ & $7.68 \%$ & $5.30 \%$ & $7.64 \%$ & $6.77 \%$ \\
\hline Sharpe ratio & 0.3678 & 0.3544 & 0.7013 & 0.3602 & 0.3228 & 0.7016 \\
\hline Final wealth & 4.2751 & 3.4621 & 5.7563 & 2.8048 & 3.153 & 5.0512 \\
\hline
\end{tabular}

TABLE 7: Performance statistics of REDP strategy with different drawdown limit.

\begin{tabular}{|c|c|c|c|c|c|c|c|c|}
\hline \multirow{2}{*}{ Statistics $/ \delta$} & \multicolumn{4}{|c|}{ Without correlation coefficient } & \multicolumn{4}{|c|}{ With correlation coefficient } \\
\hline & $1 / 3$ & 0.25 & 0.2 & 0.15 & $1 / 3$ & 0.25 & 0.2 & 0.15 \\
\hline Max REDD & $11.87 \%$ & $8.89 \%$ & $9.00 \%$ & $10.91 \%$ & $7.96 \%$ & $5.20 \%$ & $4.41 \%$ & $4.40 \%$ \\
\hline Mean REDD & $2.24 \%$ & $1.46 \%$ & $1.12 \%$ & $0.97 \%$ & $1.99 \%$ & $1.18 \%$ & $0.77 \%$ & $0.44 \%$ \\
\hline Maximum drawdown & $11.85 \%$ & $8.21 \%$ & $8.32 \%$ & $9.33 \%$ & $8.13 \%$ & $4.39 \%$ & $3.62 \%$ & $3.61 \%$ \\
\hline Annualized return & $8.28 \%$ & $7.47 \%$ & $6.78 \%$ & $5.87 \%$ & $7.64 \%$ & $7.20 \%$ & $6.68 \%$ & $6.04 \%$ \\
\hline Annualized return S.E. & $7.68 \%$ & $5.79 \%$ & $4.87 \%$ & $4.09 \%$ & $6.77 \%$ & $5.16 \%$ & $4.04 \%$ & $3.00 \%$ \\
\hline Sharpe ratio & 0.7013 & 0.7908 & 0.7988 & 0.7285 & 0.7016 & 0.834 & 0.9373 & 1.0471 \\
\hline Final wealth & 5.7563 & 4.8812 & 4.2336 & 3.5104 & 5.0512 & 4.6116 & 4.1461 & 3.6301 \\
\hline
\end{tabular}

has a stable increase in portfolio return and efficient control of the investment risk.

The comparison between the two cases, with and without correlation coefficient, shows that introducing correlation coefficient into the three strategies would reduce the portfolio return (both annualized return and final wealth). For $Y Z$ strategy, considering correlation coefficient, the max REDD and maximum drawdown go down at the expense of $2 \%$ drop in the annualized return. For the RS strategy, including correlation coefficient leads to worse performance than the uncorrelated one. As for the REDP strategy, the annualized return is just $0.64 \%$ lower than that of the uncorrelated one, and the max REDD reduces by $3.91 \%$. Also, the Sharpe ratio is slightly improved importing the correlation coefficient into the REDP strategy.

Figures 1 and 2 clearly demonstrate the outperformance of the REDP strategy. The portfolio wealth of the REDP strategy exceeds that of the risky asset SPTR, while the portfolio wealth of the other two strategies is lower than that of SPTR.

In the uncorrelated case, the return of $Y Z$ strategy goes up and down, which may cause larger drawdown and REDD than the RS strategy and REDP strategy (max REDD and maximum drawdown are showed in Table 6). By importing the correlation coefficient into $Y Z$ strategy, the wealth curve becomes more stable in Figure 2 than in Figure 1.

From Figure 2, the increasing speed of the return of the REDP strategy slows down when the risky assets go down, because these two risky assets drop together and the high correlation coefficient will reduce the investment proportions. Besides, the high REDD of risky asset would reduce the capital invested in that asset in order to reduce risk. However, the portfolio wealth of the REDP strategy increases steadily despite the slump of risky assets in year 2009. Using the $\mathrm{REDD}_{P}$ of risky asset instead of the REDD of the portfolio can further reduce the risk and make the portfolio adjustment much flexible to different risky assets.

In a word, the empirical results indicate that REDP strategy performs better than YZ strategy and RS strategy.

5.3. REDP Strategy with Different Drawdown Limit. Since different investors have different risk preference, we further study the REDP strategy with different drawdown limit $\delta$. The cases of two risky assets with or without correlation are also compared in this part. The drawdown limit $\delta$ is set to be $1 / 3,0.25,0.2$, and 0.15 . The empirical results are presented in Table 7 .

For different drawdown limits, all of the max REDD and maximum drawdown in Table 7 are successfully controlled under the given limit. When considering correlation between the two risky assets, the max REDD, the maximum drawdown, and the annualized return are lower than without considering correlation coefficient. Besides, the Sharpe ratio is higher than the uncorrelated one.

We may notice that, except for the uncorrelated case of $\delta=0.15$, all of the max REDD, maximum drawdown, and annualized return go down with the decrease of the drawdown limit $\delta$, and the Sharpe ratio goes up accordingly. It indicates that a smaller drawdown limit leads to lower risk, but the max REDD has a limit on the degree of reduction. Moreover, the portfolio with a smaller drawdown limit produces a lower return. Therefore, it is very flexible for investors to choose different drawdown limit to achieve their risk and return objectives.

There is no doubt that the $\mathrm{REDD}_{P}$ of the risky asset can better adjust the capital invested in the risky asset, because 


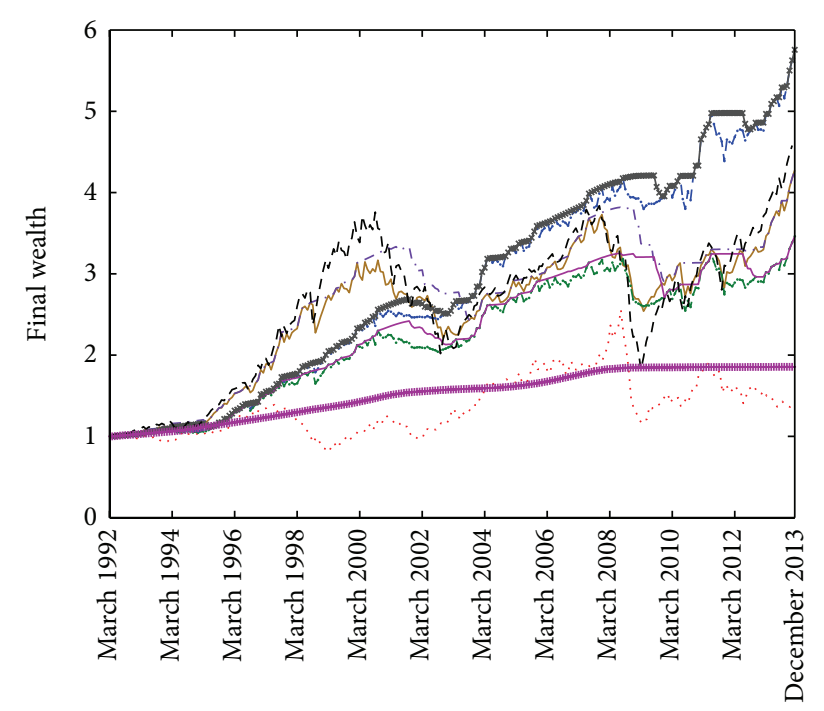

Time

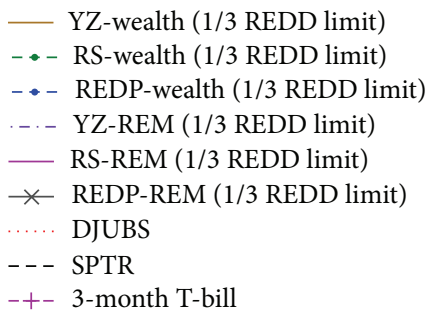

FIGURE 1: Portfolio wealth of two risky assets' investment without correlation coefficient.

$\mathrm{REDD}_{P}$ reflects the fluctuation of the risky asset itself. By using the $\mathrm{REDD}_{P}$ of the risky asset instead of the REDD of the portfolio, the REDP strategy can further reduce risk and produce much return. Besides, importing the rolling estimation of both Sharpe ratio and correlated coefficient into the REDP strategy is much more available and practical in the investment activities.

\section{Conclusion}

This paper proposes an alternative strategy called " $R S$ strategy" to YZ strategy that replaces the constant Sharpe ratio with the rolling Sharpe ratio in computing the allocation proportion. Besides, we further propose a novel strategy named "REDP strategy" which substitutes the rolling economic drawdown of the risky asset for the rolling economic drawdown of the portfolio.

We demonstrate the simulation test of single risky asset market and two-risky-asset market and verify that the $Y Z$ strategy, RS strategy, and REDP strategy succeed in controlling the max REDD of portfolio under a given drawdown limit. In addition, the simulation test results prove that the REDP strategy outperforms the other two strategies significantly. An empirical research on the comparison between the performances of the three strategies is performed by using US market data of SPTR, DJUBS, and 3-month T-bill from

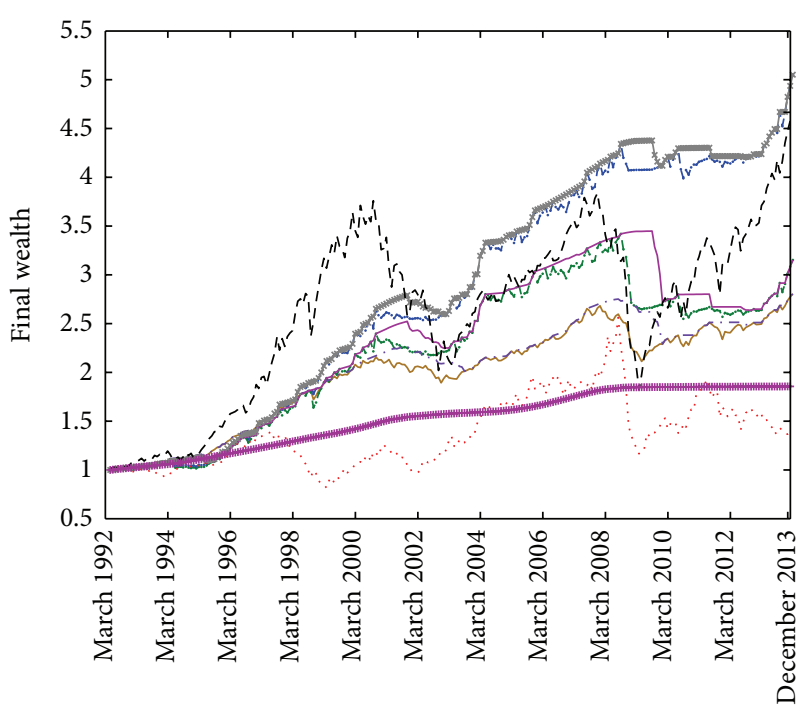

Time

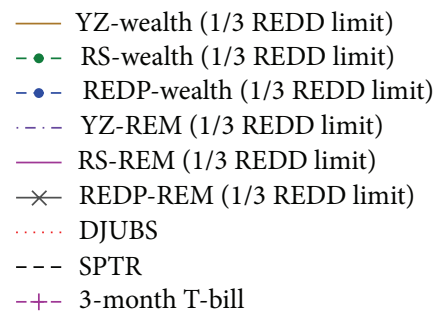

FIGURE 2: Portfolio wealth of two risky assets' investment with correlation coefficient.

January 1991 to December 2013. The empirical results also show that the REDP strategy ensures the maximum economic drawdown to stay within the drawdown limit. The comparison results indicate that the REDP strategy outperforms both $Y Z$ strategy and RS strategy at return and risk. Moreover, the proposed REDP strategy enables investors to set a drawdown limit to achieve their risk and return objectives. With a bigger drawdown limit, the portfolio can produce a higher return. In conclusion, the REDP strategy is the best among these three strategies.

In this paper, the dynamic REDD, Sharpe ratio, and correlation coefficient are estimated through a rolling time window with a constant length. However, the length of the rolling window may vary from time to time. Further research could be done in setting up dynamic predicting models based on the historical data to forecast the REDD, Sharpe ratio, and the correlation coefficient in the next period. Applying the predicted variables to adjust the investment weight could further enhance the investment performance. And a study on the mathematical property of the REDP strategy is also needed in the future.

\section{Conflict of Interests}

The authors declare that there is no conflict of interests regarding the publication of this paper. 


\section{Acknowledgments}

The authors would like to acknowledge the financial support from the National Natural Science Foundation of China (no. 71171086), Guangdong Universities Humanities and Social Science Key Research Base project (2012JDXM 0006), and the Fundamental Research Funds for the Central Universities (no. 2013ZM0120 and no. 2013X2D10).

\section{References}

[1] S. Grossman and Z. Zhou, "Optimal investment strategies for controlling draw-downs," Mathematical Finance, vol. 3, no. 3, pp. 241-276, 1993.

[2] A. Chekhlov, S. Uryasev, and M. Zabarankin, "Drawdown measure in portfolio optimization," International Journal of Theoretical and Applied Finance, vol. 8, no. 1, pp. 13-58, 2005.

[3] H. Markowitz, "Portfolio selection," Journal of Finance, vol. 7, no. 1, pp. 77-91, 1952.

[4] D. B. Brown and J. E. Smith, "Dynamic portfolio optimization with transaction costs: heuristics and dual bounds," Management Science, vol. 57, no. 10, pp. 1752-1770, 2011.

[5] L. Zhang and Z. Li, "Multi-period mean-variance portfolio selection with uncertain time horizon when returns are serially correlated," Mathematical Problems in Engineering, vol. 2012, Article ID 216891, 17 pages, 2012.

[6] H. Guo, B. Sun, H. R. Karimi, Y. Ge, and W. Jin, "Fuzzy investment portfolio selection models based on interval analysis approach," Mathematical Problems in Engineering, vol. 2012, Article ID 628295, 15 pages, 2012.

[7] F. J. DiTraglia and J. R. Gerlach, "Portfolio selection: an extreme value approach," Journal of Banking and Finance, vol. 37, no. 2, pp. 305-323, 2013.

[8] J. Cvitanic and I. Karatzas, "On portfolio optimization under drawdown constraints," IMA Lecture Notes in Mathematics and Applications, vol. 65, pp. 77-88, 1995.

[9] J. Sekine, "A note on long-term optimal portfolios under drawdown constraints," Advances in Applied Probability, vol. 38, no. 3, pp. 673-692, 2006.

[10] H. Nagai, "Optimal strategies for risk-sensitive portfolio optimization problems for general factor models," SIAM Journal on Control and Optimization, vol. 41, no. 6, pp. 1779-1800, 2003.

[11] H. Kaise and S. J. Sheu, "Risk sensitive optimal investment: solutions of the dynamical programming equation," Contemporary Mathematics, vol. 351, pp. 217-230, 2004.

[12] L. Pospisil and J. Vecer, "Portfolio sensitivity to changes in the maximum and the maximum drawdown," Quantitative Finance, vol. 10, no. 6, pp. 617-627, 2010.

[13] Z. Yang and L. Zhong, "Towards optimal portfolio strategy to control maximum drawdown: the case of risk based dynamic asset allocation," China Finance Review International, vol. 3, no. 2, pp. 131-163, 2013. 


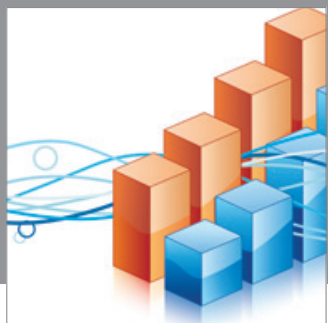

Advances in

Operations Research

mansans

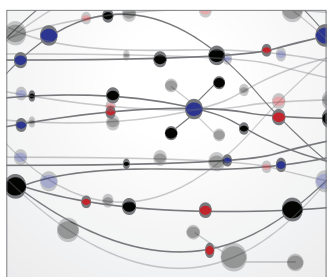

The Scientific World Journal
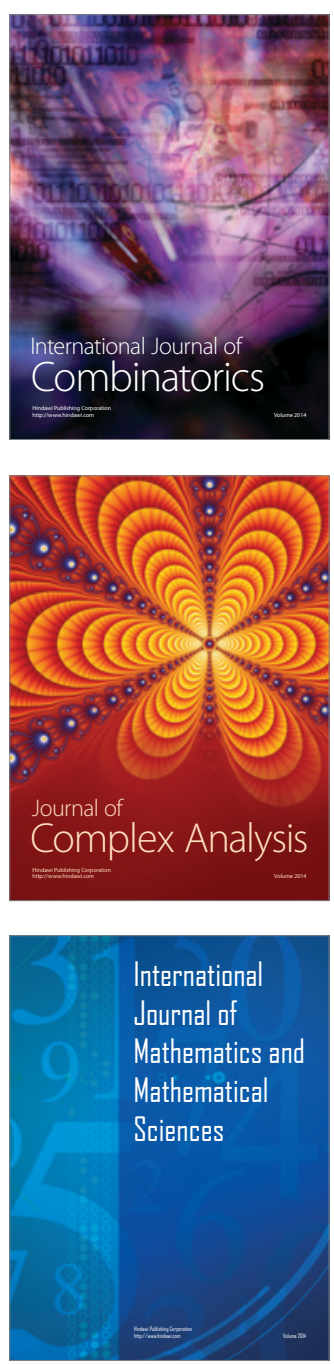
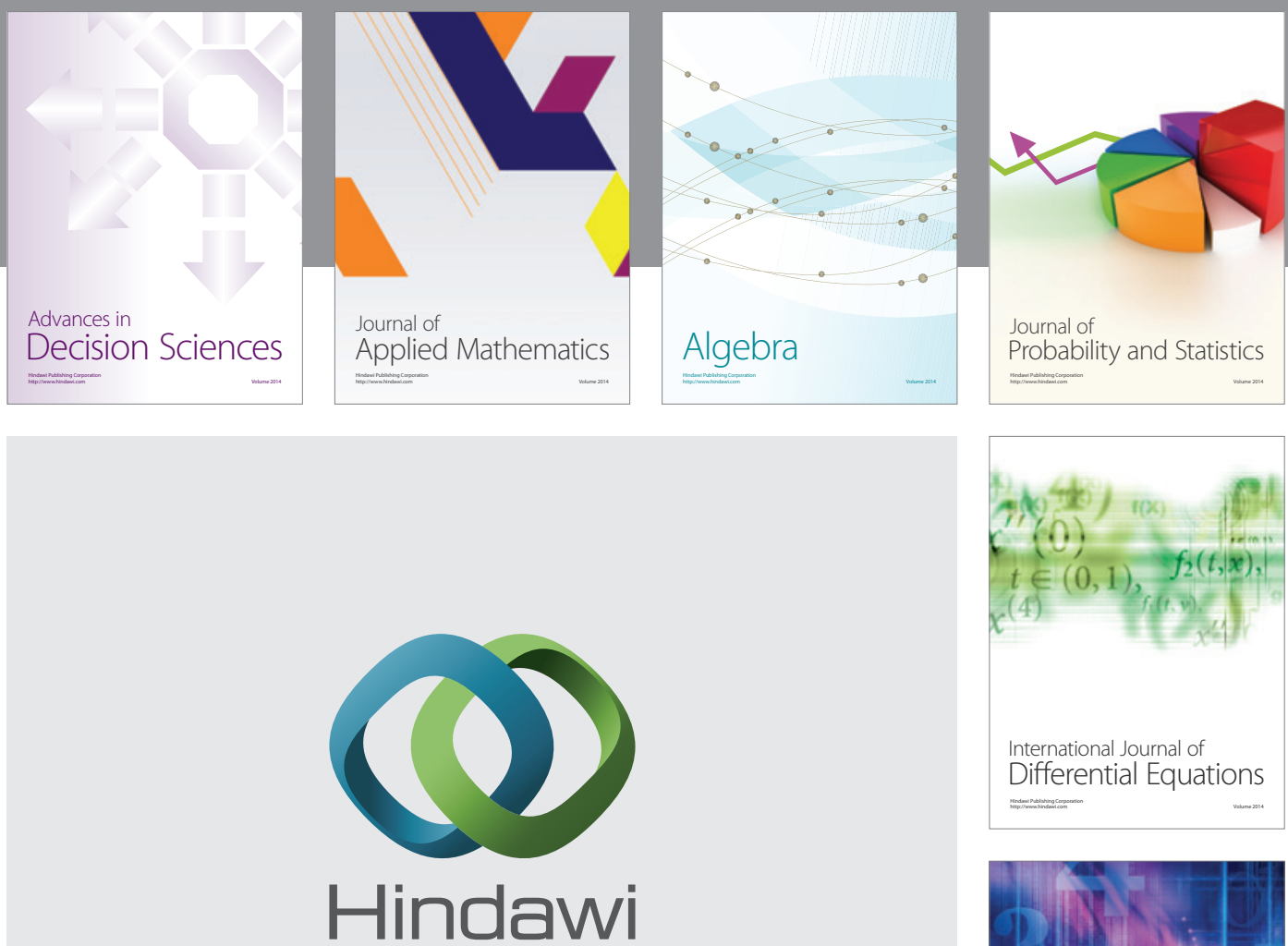

Submit your manuscripts at http://www.hindawi.com
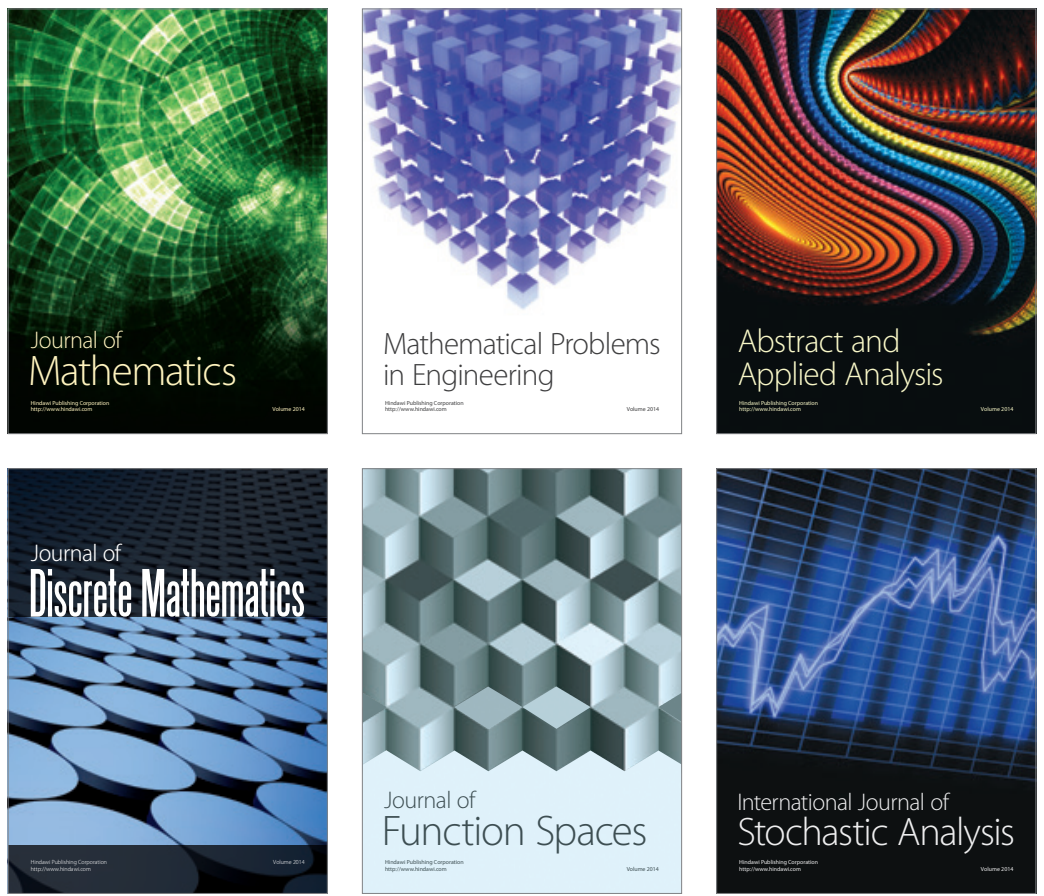

Journal of

Function Spaces

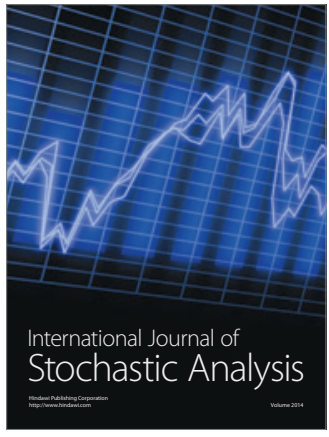

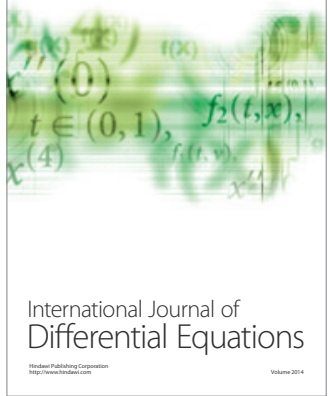
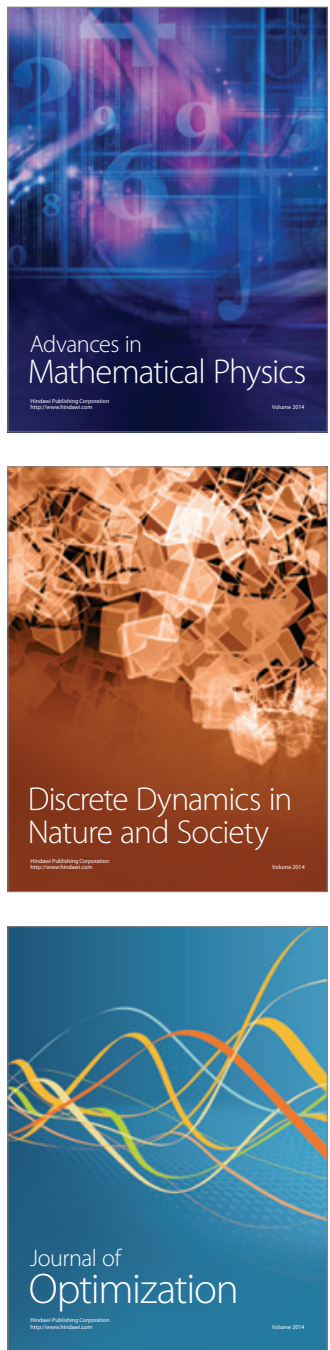